\title{
Inducción a las Matemáticas Empleando Objetos de Aprendizaje y Diseño Instruccional
}

\section{Induction to Mathematics Using Learning and Design Objects}

\author{
Matilde Reyes-Fuentes ${ }^{a}$, Silvia P. Ambrocio-Cruz ${ }^{b}$, Daniel Vélez-Díaz ${ }^{c}$
}

\begin{abstract}
:
Online learning is a global way and implemented today. Modern teaching techniques are of vital importance for the teacher and the student, due to their constant training in technological tools and the current situation in which we find ourselves, to have an updated educational system requires technologies with increasing use of digital games and applied sciences in a learning environment, affecting the student in their training. The greatest challenge for a professor is to prepare his content more quickly and to successfully conclude the class session at a distance and with a large number of distractions. Each group you teach has different personalities, abilities, and learning preferences. The next generations of students have a tremendous advance over the digital age, enabling the teacher to create a variety of activities that maintain the interest in learning. To obtain a learning activity, it must be well designed for the learning process to be effective and with its respective implementation. Learning based on the integration of pre-established plugins plays an important role in the teacher, allowing participation, interaction and collaboration in a classroom and delivering better results. The proposal presented in this document allows the development of didactic and practical material for new students in mathematical skills, allowing them to reinforce their skills in possible subjects of their professional career.
\end{abstract}

Keywords:

Learning, learning environments, technological tools, modern educational system, training.

\section{Resumen:}

El aprendizaje en línea es una forma global y puesta en práctica hoy en día. Las técnicas de enseñanza moderna son de vital importancia para el docente y el estudiante, por su constante capacitación en herramientas tecnológicas y la situación actual en la cual nos encontramos, para contar un sistema educativo actualizado se requiere de tecnologías con uso creciente de juegos digitales y ciencias aplicadas en un entorno de aprendizaje, afectando al estudiante en su formación. El mayor reto que posee un catedrático es la preparación de su contenido con mayor rapidez y concluir exitosamente la sesión de clase a distancia y con gran cantidad de distractores. Cada grupo al que imparte clase posee diferentes personalidades, capacidades y preferencias de aprendizaje. Las generaciones próximas de estudiantes poseen un avance enorme sobre la era digital, propiciando al maestro crear una variedad de actividades que mantengan el interés por aprender. Para obtener una actividad de aprendizaje, debe estar bien diseñada para que el proceso de aprendizaje sea efectivo y con su respectiva implementación. El aprendizaje basado en la integración de complementos preestablecidos juega un papel importante en el profesor, lo que permite que exista la participación, interacción y colaboración en un salón de clases y entregando mejores resultados. La propuesta realizada que presenta este documento permite la elaboración de un material didáctico y práctico para los estudiantes de nuevo ingreso en habilidades matemáticas permitiendo reforzar sus competencias en posibles asignaturas de su carrera profesional.

\section{Palabras Clave:}

Aprendizaje, entornos de aprendizaje, herramientas tecnológicas, sistema educativo moderno, capacitación.

\footnotetext{
a Autor de Correspondencia, Universidad Autónoma del Estado de Hidalgo, Escuela Superior de Tlahuelilpan, https://orcid.org/0000-00017902-2840,Email: matilde_reyes@uaeh.edu.mx

b Universidad Autónoma del Estado de Hidalgo, Escuela Superior de Tlahuelilpan, https://orcid.org/0000-0001-8452-487X, Email: silviap@uaeh.edu.mx

Universidad Autónoma del Estado de Hidalgo, Escuela Superior de Tlahuelilpan, https://orcid.org/0000-0001-6488-2960, Email: daniel@uaeh.edu.mx
} 


\section{Introducción}

A través de los avances tecnológicos de vanguardia y un sistema educativo más globalizado, las prácticas de aprendizaje han ido incorporando nuevas herramientas con un enfoque en el uso de tecnologías y se ha vuelto casi una obligación en diferentes áreas laborales. Los actuales modelos educativos presentan desafíos importantes, uno de ellos es la innovación el proceso de enseñanza-aprendizaje, considerando que la clase tradicional supone a nuevos cambios metodológicos y actitudinales. Otro es la independencia del tiempo y el espacio, con un mejor acceso a la educación a través de medios electrónicos, material establecido en línea y la educación semipresencial (Blended learning). Por lo que el aprendizaje a través de plataformas virtuales permite la innovación entre la teoría-práctica para la formación de los estudiantes y generando un significativo desempeño. Considerando que el rol del profesor incluye recursos tecnológicos, metodologías innovadoras y modelos de diseño instruccional que integren las herramientas de Tecnologías de la Información. Cabe resaltar que los procesos pedagógicos necesitan de estas herramientas para cumplir sus objetivos y no quedarse relegados con un modelo educativo menos enriquecedor y sin capacidad de adaptarse. Generando beneficios en un área específica: Educación. Esto con el fin de superar las barreras y limitaciones de tiempo establecidas en la educación base, seleccionando un nuevo ritmo de enseñanza, con mejores servicios y calidad en materiales personalizados, así como un seguimiento, autoevaluación y monitorización en el rendimiento del alumno, convirtiendo a este como un agente activo entre la práctica docente y el aprendizaje electrónico. Manifestando las características anteriores, se pueden implementar en un solo modelo educativo a través de una mezcla de actividades en redes digitales y que cada sesión de clase presente un diseño educativo bien balanceado.

El presente trabajo describe cómo se va a llevar a cabo la implementación de un curso en la plataforma virtual Moodle del área Académica de Tecnologías de la Información, con su respectiva plantilla y plugins para brindar una mejor usabilidad tanto al docente como a los alumnos.

Una de las causas principales que se tomaron en cuenta para llevar a cabo este proyecto, es el bajo desempeño que han presentado algunos alumnos de nuevo ingreso en sus habilidades matemáticas. Teniendo como resultado algunas dificultades a la hora de tomar materias de lógica, aritmética, álgebra, entre otras

Para combatir este problema, se busca crear un curso propedéutico con el cual adquieran o refuercen habilidades de aritmética y álgebra básica, haciendo uso de la plataforma virtual, así como de herramientas adicionales (PhET Simulations, H5P, Juegos, entre otros). Cabe mencionar, que diversas instituciones tanto del país como del mundo hacen uso de estas herramientas.

\section{Metodología y Procedimientos}

Las tecnologías en el aspecto educativo permiten que se les observe en cualquier interés particular.

\section{Metodología ADDIE}

ADDIE es el acrónimo de las 5 fases del sistema instruccional. [1]. El concepto de diseño instruccional se remonta a la década de 1950. Pero no fue hasta 1975 que se diseñó ADDIE, establecida por la Universidad del Estatal de Florida. Consta del Análisis (Analyze), diseño (Design, desarrollo (Development), implementación (Implementation) y evaluación (Evaluation). Es un plan estratégico para el diseño del curso y para desarrollar programas educativos y de capacitación. Los educadores, diseñadores de instrucción y desarrolladores de capacitación encuentran este enfoque muy útil porque tener etapas definidas claramente facilita la implementación de herramientas de capacitación efectivas. Como modelo de identificación, ADDIE ha encontrado una amplia aceptación y uso. Debido a la estructura jerárquica de los pasos, uno tenía que completar el proceso de forma lineal, completando una fase antes de comenzar la siguiente.

Los practicantes a lo largo de los años han realizado varias revisiones en las etapas de la versión jerárquica original. Esto ha hecho que el modelo sea más interactivo y dinámico. Fue a mediados de la década de 1980 cuando apareció la versión similar a la versión actual. Hoy en día, la influencia del método ADDIE se puede ver en la mayoría de los modelos de ID que se utilizan. Estos 5 componentes se presentan de la siguiente manera:

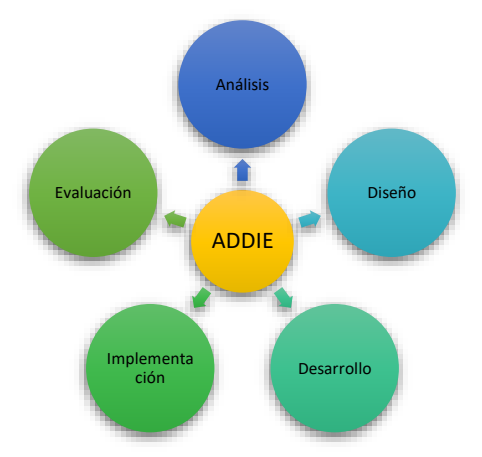

\section{Análisis}

Puede considerarse como la "Etapa de fijación de objetivos". El enfoque del diseñador en esta fase está en el público objetivo. También es aquí donde el programa coincide con el nivel de habilidad e inteligencia que muestra cada estudiante / participante. Esto es para 
asegurar que lo que ya saben no se duplicará, y que el enfoque se centrará en temas y lecciones que los estudiantes aún tienen que explorar y aprender. En esta fase, los instructores distinguen entre lo que los estudiantes ya saben y lo que deberían saber después de completar el curso.

Se deben utilizar varios componentes clave para garantizar que el análisis sea exhaustivo. Los textos y documentos del curso, los planes de estudio y el internet deben ser empleados. Con la ayuda de materiales en línea, como cursos en línea, se puede determinar una estructura como la guía principal para el programa de estudios.

\section{Diseño}

Determina todos los objetivos, las herramientas que se utilizarán para medir el rendimiento, diversas pruebas, análisis de temas, planificación y recursos. En la fase de diseño, el enfoque se centra en los objetivos de aprendizaje, el contenido, el análisis de la materia, el ejercicio, la planificación de la lección, los instrumentos de evaluación utilizados y la selección de medios.

El enfoque en esta fase debe ser sistemático con un proceso lógico y ordenado de identificación, desarrollo y evaluación de las estrategias planificadas que apuntan al logro de los objetivos del proyecto. Debe seguir un conjunto de reglas muy específico, y cada elemento del plan de diseño instruccional debe ejecutarse con atención al detalle. Ser un factor estricto para los detalles es crucial para el éxito de la etapa de diseño. Este enfoque sistemático asegura que todo cae dentro de una estrategia racional y planificada, o un conjunto de estrategias, que tiene el objetivo final de alcanzar los objetivos del proyecto.

\section{Desarrollo}

Comienza la producción y prueba de la metodología utilizada en el proyecto. En esta etapa, los diseñadores hacen uso de los datos recopilados de las dos etapas anteriores y usan esta información para crear un programa que transmitirá lo que se debe enseñar a los participantes. Si las dos etapas anteriores requerían planificación y lluvia de ideas, la etapa de Desarrollo se trata de ponerlo en práctica. Esta fase incluye tres tareas, a saber, redacción, producción y evaluación. Por lo tanto, implica la creación y prueba de los resultados del aprendizaje.

\section{Implementación}

Refleja la modificación continua del programa para garantizar la máxima eficiencia y obtener resultados positivos. Aquí es donde los ID se esfuerzan por rediseñar, actualizar y editar el curso para garantizar que se pueda entregar de manera efectiva. "Procedimiento" es la palabra clave aquí. Gran parte del trabajo real se realiza aquí, ya que las identificaciones y los estudiantes trabajan de la mano para capacitarse en nuevas herramientas, de modo que el diseño pueda evaluarse continuamente para una mejora adicional.

La evaluación del diseño se realiza en la fase de implementación. Los diseñadores desempeñan un papel muy activo en esta etapa, que es crucial para el éxito del proyecto. Los desarrolladores deben analizar, rediseñar y mejorar constantemente el producto para garantizar la entrega efectiva del producto. El monitoreo meticuloso es imprescindible. En esta fase se realiza una evaluación adecuada del producto, curso o programa, con las revisiones necesarias y oportunas. Cuando los instructores y los alumnos contribuyen activamente durante el proceso de implementación, se pueden realizar modificaciones instantáneas en el proyecto, haciendo que el programa sea más efectivo y exitoso.

\section{Evaluación}

Es la etapa en la que el proyecto está siendo sometido a pruebas finales meticulosas sobre qué, cómo, por qué, cuándo de las cosas que se lograron (o no se lograron) de todo el proyecto. Esta fase se puede dividir en dos partes: formativa y sumativa. La evaluación inicial realmente ocurre durante la etapa de desarrollo. La fase de formación ocurre mientras los estudiantes y las identificaciones están realizando el estudio, mientras que la parte sumativa se produce al final del programa. El objetivo principal de la etapa de evaluación es determinar si se han cumplido los objetivos y establecer qué se requerirá para avanzar con el fin de promover la eficiencia y la tasa de éxito del proyecto.

\section{DESARROLLO}

\section{Identificación de características moodle 3.2.1}

De acuerdo con la información que proporciona la plataforma y su versión "3.2 se enfoca en mejorar la experiencia del usuario y en la Accesibilidad" [2] con énfasis en los roles:

- Para profesores.

\begin{tabular}{|l|l|}
\hline \multicolumn{1}{|c|}{ Característica } & \multicolumn{1}{c|}{ Descripción } \\
\hline $\begin{array}{l}\text { 1.Mejor navegación } \\
\text { con Boost con } \\
\text { Bootstrap 4 }\end{array}$ & $\begin{array}{l}\text { Aplicación de un diseño } \\
\text { web responsivo para } \\
\text { mejorar la navegación en } \\
\text { cursos. [3] }\end{array}$ \\
\hline $\begin{array}{l}\text { 2.Menús con } \\
\text { engranes }\end{array}$ & $\begin{array}{l}\text { Modernizar la experiencia } \\
\text { en la edición de cursos. } \\
\text { [2] }\end{array}$ \\
\hline $\begin{array}{l}\text { 3.Reproductor de } \\
\text { medios }\end{array}$ & $\begin{array}{l}\text { Soporte a través de } \\
\text { Video.JS para varios } \\
\text { dispositivos y } \\
\text { navegadores compatibles } \\
\text { de Escritorio: Cromo, }\end{array}$ \\
& $\begin{array}{l}\text { Firefox, Safari, Borde e } \\
\text { Internet Explorer. } \\
\text { Móvil: MobileSafari y } \\
\text { Google Chrome. [4] }\end{array}$ \\
\hline
\end{tabular}




\begin{tabular}{|l|l|}
\hline 4.Gráficas interactivas & $\begin{array}{l}\text { Los reportes se muestran } \\
\text { de forma dinámica a cada } \\
\text { participante del curso. [5] }\end{array}$ \\
\hline $\begin{array}{l}\text { 5.Anulación de } \\
\text { actividad }\end{array}$ & $\begin{array}{l}\text { Permite cambiar la fecha } \\
\text { límite de una tarea para } \\
\text { varios individuos o grupos. } \\
{[6]}\end{array}$ \\
\hline
\end{tabular}

- Para todos los usuarios.

\begin{tabular}{|l|l|}
\hline Característica & Descripción \\
\hline 1.Mensajería y alertas & $\begin{array}{l}\text { Acceso rápido a } \\
\text { notificaciones y } \\
\text { mensajes desde } \\
\text { cualquier lugar. [7] }\end{array}$ \\
\hline 2.A.P.P. Mobile & $\begin{array}{l}\text { Permite soporte a } \\
\text { exámenes fuera de línea } \\
\text { y configuraciones en } \\
\text { nivel administrador a } \\
\text { través de la aplicación. } \\
{[8],[9]}\end{array}$ \\
\hline
\end{tabular}

\section{Recopilación de información de acuerdo con el} temario propuesto del curso

Aritmética - Operaciones Básicas. De acuerdo con la información proporcionada, son cuatro operaciones matemáticas: la suma, la resta, la multiplicación y la división. Las operaciones básicas de las matemáticas nos sirven mucho para la vida cotidiana. [10]

3. Adición de plugins de interacción en el aula virtual A partir de la página oficial de la plataforma, podemos observar para la compatibilidad de la versión 3.2.1 en sus elementos adicionales y para el uso de actividades, los siguientes módulos:

- Geogebra

- Álgebra

- H5P

- E-Voting

- Juegos

\section{Identificación de software complementario para material didáctico}

Para el proceso de selección, en el caso del docente, se obtuvieron los elementos como:

- Kahoot!. El aprendizaje social que promueve esta plataforma es basada en juegos y es gratuita para profesores. Permite crear cuestionarios de opción múltiple, incluyendo el agregado de videos, imágenes y diagramas a las preguntas para amplificar el compromiso. Fomenta la participación grupal a través de cada alumno con su propio dispositivo sin poseer una cuenta dentro de la plataforma. [11]

- GoConqr. Es una plataforma de aprendizaje social y académica en la que se le pueden proporcionar tanto como al docente y estudiantes compartir los objetos de aprendizaje. De igual forma, posee la creación de mapas mentales, juegos de preguntas y respuestas, diapositivas y tarjetas para memorizar.
Lo que favorece a esta plataforma es su soporte bajo dispositivos iOS y Android, lo que permite trabajar desde cualquier parte o considerar para el estudiante, su propio bloc de notas para cualquier clase. Cabe resaltar que si requieren de mayor almacenamiento para compartir sus recursos entre profesor-alumno, sólo soporta hasta 50 M.B. en archivos y teniendo un plan de pago colectivo o individual y combina los beneficios de potentes herramientas de aprendizaje junto con el apoyo de una comunidad de estudiantes y profesores activa. [12]

- PhET Simulations. A través de la Universidad de Colorado, las simulaciones de PhET se basan en simulaciones interactivas gratuitas de matemáticas y otras ciencias. Se basan en la investigación educativa extensiva e involucran a los estudiantes mediante un ambiente intuitivo y similar a un juego, en donde aprenden explorando y descubriendo [13]. Estos simuladores se eligieron con el propósito de proporcionar al alumno como recurso externo en el curso.

- ThatQuiz. Se basa en diferentes ámbitos matemáticos: Desde fracciones, conceptos básicos, geometría, etc. Posee un ambiente multilenguaje para mayor y no requiere que un alumno posea una cuenta para utilizarlo. Es un sitio bastante sencillo, se tomarán las aplicaciones en el tema de expresiones algebraicas. [14]

- Calcu World. Posee múltiples calculadoras matemáticas para la implementación a través de frames que faciliten el aprendizaje como la calculadora de números primos, tablas de multiplicar, porcentajes, radicación, división, fracciones, entre otros. Así mismo, tiene para otras áreas de interés. [15]

\section{Apariencia y navegación de plantilla Lambda}

- Compatibilidad Moodle 3.2.1-Lambda. Contiene soporte multilenguaje, adaptaciones de bloques con personalizado H.T.M.L., permite combinaciones de tipografías a través del C.S.S., cuenta con enlace adicional para el acceso por autentificación de cuentas Facebook, Google, Microsoft y con amplia flexibilidad en el diseño. A continuación, se presenta la ficha técnica del tema a implementar con la versión 3.2.1 de la plataforma:

\begin{tabular}{|l|l|}
\hline \multicolumn{1}{|c|}{ Característica } & \multicolumn{1}{c|}{ Descripción } \\
\hline Última versión & 17 de Junio de 2019 \\
\hline Creación & 28 de noviembre de 2019 \\
\hline Compatible con & IE8, IE9, IE10, IE11, \\
navegadores & Firefox, Safari, Opera, \\
& Chrome, Edge \\
\hline Versión(es) moodle & Moodle 3.5, Moodle 3.4, \\
compatibles & Moodle 3.3.x, Moodle \\
& $3.2 . x$, Moodle 3.1.x \\
& Moodle 3.0.x, Moodle \\
\hline
\end{tabular}




\begin{tabular}{|l|l|}
\hline & 2.9.x, Moodle 2.8.x, \\
& Moodle 2.7.x, Moodle \\
& 2.6.x, Moodle 2.5.x \\
\hline Lenguajes soportados & P.H.P., C.S.S. y J.S. \\
\hline$[13]$ & \\
\hline
\end{tabular}

\section{Características de los plugins para interacción}

- GeoGebra. Para el ámbito de ciencias exactas se reconoce que esta herramienta es un potente software de matemáticas para todo nivel educativo. Reúne dinámicamente geometría, álgebra, estadística y cálculo en registros gráficos, de análisis y de organización en hojas de cálculo. GeoGebra, con su libre agilidad de uso, congrega a una comunidad vital y en crecimiento. En todo el mundo, millones de entusiastas lo adoptan y comparten diseños y aplicaciones de GeoGebra. Dinamiza el estudio. Armonizando lo experimental y lo conceptual para experimentar una organización didáctica y disciplinar que cruza matemática, ciencias, ingeniería y tecnología [14]. Dentro de las características que posee como módulo en la plataforma de Moodle se encuentran involucradas en las actividades:

1. Permite incrustar fácilmente las actividades de GeoGebra en algunos cursos de Moodle.

2. Facilita el rastreo de los estudiantes porque almacena la puntuación, la fecha, la duración y la construcción de cada uno de los intentos realizados por los usuarios.

3. Los estudiantes pueden guardar el estado de las actividades para continuarlas más tarde. [15]

4. El complemento de tipo de pregunta de GeoGebra permite a los maestros configurar preguntas que pueden resolverse y verificarse automáticamente utilizando GeoGebra. el estudiante tiene que resolver la tarea en GeoGebra.

a. El estado del applet se guarda en Moodle para su calificación o en caso de que haya sido posible calificar automáticamente la tarea para su revisión.

b. El tipo de pregunta admite la aleatorización de los controles deslizantes en el applet y las variables de sustitución en el texto de la pregunta con los valores de este control deslizante.

c. Si hay objetos booleanos presentes en el applet que indican la corrección de una solución, esos objetos se pueden usar para calificar automáticamente. [16]

- Álgebra. La interacción basada en esta disciplina elemental se compone con un tipo de pregunta algebraica donde las respuestas de los alumnos se tratan como una expresión algebraica y se comparan con las respuestas proporcionadas por el instructor utilizando las reglas básicas del álgebra. [17]

- Contenido Interactivo-H5P. A la hora de crear actividades o recursos para los estudiantes, el docente presenta situaciones más complejas: tiempo para el diseño y creación de objetos de aprendizaje más rápidos y eficientes, poco conocimiento con las herramientas basadas en la computación y sin actualizar, el formato más adecuado a la hora de compartirlos con sus alumnos. Un gran beneficio con H5P es que le permite compartir y reutilizar fácilmente el contenido. [18] Conforme el avance tecnológico se ha hecho más grande, llega este instrumento con una gran variedad de opciones para presentar contenido e insertar información adicional para una mejor experiencia de aprendizaje.

H5P cuenta con actividades para su creación como:

- Acordeones
- Secuencias de imágenes
- Pruebas aritméticas
- Gráficas
- Tareas para arrastrar palabras
- Cuestionarios personalizados
- Preguntas falso/verdadero
- Entre otros [19]

- E-Voting. Este módulo puede ser usado para permitir que los estudiantes puedan votar a través de su teléfono inteligente o su computadora portátil y que los resultados se muestren en un gráfico en tiempo real.

La actividad de E-Voting es ideal para captar la atención de grupos amplios. Aumenta las interacciones entre "profesores-alumnos". Por lo tanto, es compatible con un tipo de actividad de clase volteada.

La votación electrónica se puede utilizar en clase para verificar los conocimientos al final de un curso o para animar presentaciones teóricas. [20]

- Juegos. A partir de un cuestionario, glosario de términos o banco de preguntas pueden establecerse los siguientes juegos como recursos:

$$
\begin{array}{ll}
\text { - } & \text { Hangman } \\
\text { - } & \text { Crucigrama } \\
- & \text { Cryptex } \\
- & \text { Millonario } \\
- & \text { Sudoku } \\
- & \text { Serpientes y Escaleras } \\
- & \text { La imagen oculta } \\
- & \text { Libro con preguntas [21] }
\end{array}
$$




\section{Resultados}

Como resultado se tiene la implementación del curso en la plataforma Moodle con el contenido del curso de habilidad Matemática en donde se implementó la plataforma Lambda y los plugins mencionados.

Navegación en el curso

Para la creación del curso y su navegación se establecieron los siguientes apartados en formato de rejilla:

- Bienvenida al curso

Consiste en proporcionar al alumno los datos generales del docente para mayor duda o aclaración:

- Nombre del catedrático

- Correo electrónico

- Teléfono celular

- Ubicación de su cubículo

- Trayectoria

- Horario de asesoría

- Presentación de la asignatura

De acuerdo con el temario se establecen dos unidades que se presentan como Aritmética y Álgebra que posteriormente sus actividades estarán por separado.

\section{Aritmética}

Esta sección se implementan los recursos para los alumnos sobre las operaciones básicas de la materia, la aplicación de la ley de los signos, los números fraccionarios, múltiplos y divisores, la regla de tres y la teoría de conjuntos.

\section{Álgebra}

Se crearon objetos de aprendizaje para el uso de las expresiones algebraicas, los monomios y sus casos prácticos, los polinomios y sus operaciones, la factorización, las fracciones algebraicas incluyendo ecuaciones de primer y segundo grado.

Formatos de entrega de actividades

Se establecerán con la siguiente información:

- Nombre de la actividad

- Valor

- Fecha programada

Las modificaciones que se establecieron para la plataforma son las siguientes:

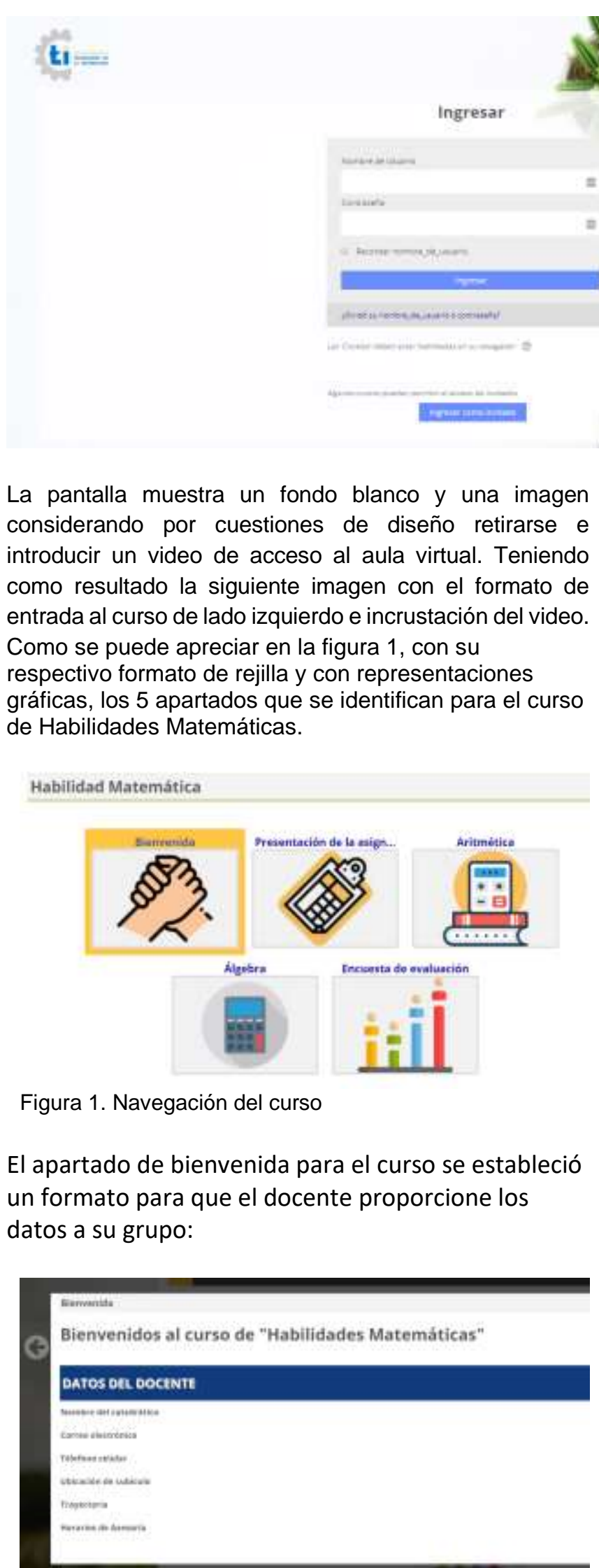

Figura 2. Formato de datos generales del docente 
Figura 7. Teoría

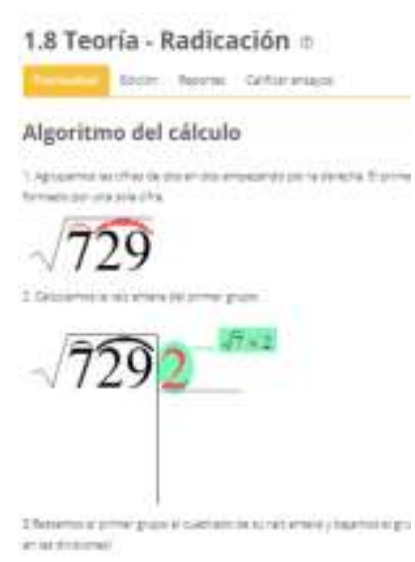

Figura 9. Ejercicio con H5P de potenciación

Figura 10. Radicales y

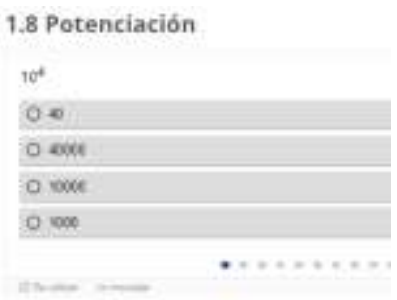

En la sección de presentación de la asignatura se define el apartado de contenido de curso para

Aritmética y Álgebra:

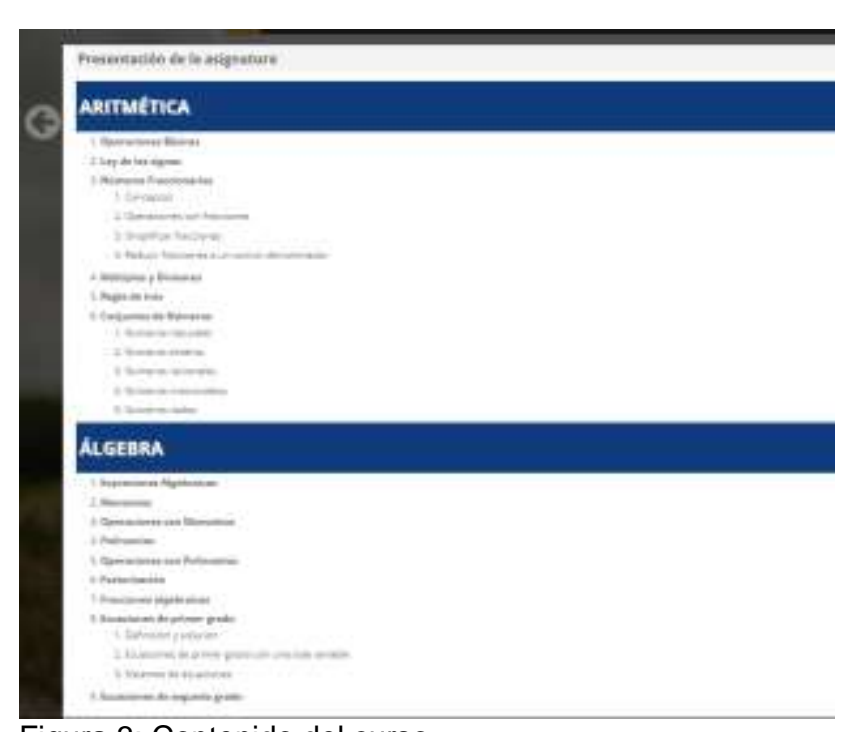

Figura 3: Contenido del curso

Figura 8. Ejercicio con H5P de radicación

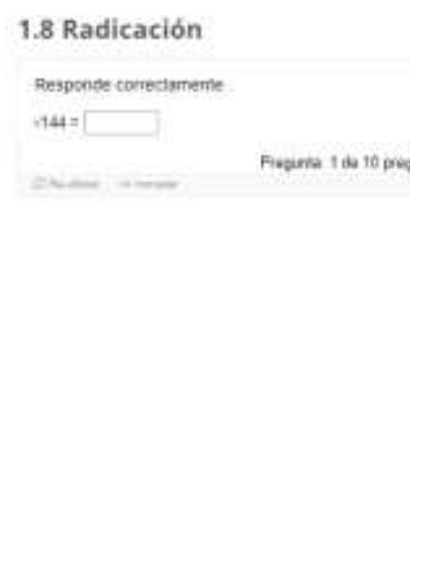

exponentes

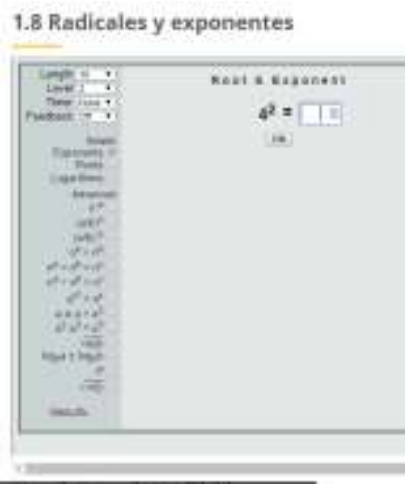

\section{Implementación de Objetos de Aprendizaje}

Para los fines prácticos, las ecuaciones y su traducción a la plataforma fue un reto debido al reconocimiento del lenguaje. Considerando que las otras herramientas que se presentan en este artículo sean otra alternativa de lo que posee la plataforma y sus herramientas adicionales para el ámbito matemático. Para el diseño de cada material, a partir de imágenes, textos y frases cortas permitieron que se lograra con satisfacción los juegos complementarios que posee cada tema y sus ejercicios. Como factor importante, el tiempo y la organización de este proyecto, se espera que la aplicación de la primera evaluación y el término del curso se encuentren los resultados positivos y significativos. Ya se diseñó la estructura del curso, se implementaron los objetos de aprendizaje y está próximo a agregar a los estudiantes de nuevo ingreso. 


\section{Referencias}

[1] S. Kurt, «Educational Technology-ADDIE Model: Instructional Design,» 29 Agosto 2017. [En línea]. Available:

https://educationaltechnology.net/the-addie-model-instructionaldesign/. [Último acceso: 26 Julio 2019].

[2] Moodle, «Nuevas características de Moodle 3.2,» 11 Noviembre 2018. [En línea]. Available:

https://docs.moodle.org/all/es/Nuevas_caracter\%C3\%ADsticas_de _Moodle_3.2. [Último acceso: 24 Junio 2019].

[3] Moodle, «New "Boost" Bootstrap 4 theme,» 27 Julio 2018. [En línea]. Available: https://tracker.moodle.org/browse/MDL-55071. [Último acceso: 24 Junio 2019].

[4] Moodle, «Introduce pluggable media players in Moodle, add VideoJS player,» 08 Agosto 2017. [En línea]. Available: https://tracker.moodle.org/browse/MDL-38158. [Último acceso: 24 Junio 2019].

[5] Moodle, «Introduce a new chart API and library,» 29 Mayo 2019. [En línea]. Available: https://tracker.moodle.org/browse/MDL54987. [Último acceso: 24 Junio 2019].

[6] Moodle, «Add user/group overrides for mod/assign,» 30 Mayo 2019. [En línea]. Available: https://tracker.moodle.org/browse/MDL-29795. [Último acceso: 24 Junio 2019].

[7] Moodle, «Messaging changes for 3.2,» 21 Mayo 2019. [En línea]. Available: https://tracker.moodle.org/browse/MDL-54682. [Último acceso: 24 Junio 2019].

[8] Moodle, «Changes in the quiz module for supporting offline quizzes in the Mobile app,»06 Diciembre 2016. [En línea]. Available: https://tracker.moodle.org/browse/MDL-53870. [Último acceso: 24 Junio 2019].

[9] Moodle, «Move mobile settings to top-level admin,»06 Diciembre 2016. [En línea]. Available:

https://tracker.moodle.org/browse/MDL-56607. [Último acceso: 24 Junio 2019].

[10] Wikipedia, «Operaciones basicas/Taller,» 1 Noviembre 2017. [En línea]. Available:

https://es.wikipedia.org/wiki/Usuario:Operaciones_basicas/Taller. [Último acceso: 09 Julio 2019].

[11] Kahoot!, 2019. [En línea]. Available: https://kahoot.com/what-iskahoot/. [Último acceso: 19 Julio 2019].

[12] GoConqr, «Aprendiendo, al modo GoConqr,» 2016. [En línea] Available: https://www.goconqr.com/es/info/sobre-nosotros/. [Último acceso: 14 Julio 2019].

[13] EnvatoMarket, «Lambda,» 2019. [En línea]. Available: https://themeforest.net/item/lambda-responsive-moodletheme/9442816. [Último acceso: 03 Julio 2019].

[14] Geogebra, «¿Qué es GeoGebra?» 2019. [En línea]. Available: https://www.geogebra.org/about. [Último acceso: 3 Julio 2019].

[15] Moodle, «Actividades: Geogebra,» 2019. [En línea]. Available: https://moodle.org/plugins/pluginversions.php?plugin=mod_geoge bra. [Último acceso: 3 Julio 2019].
[16] Moodle, «Tipos de preguntas: GeoGebra,» 2019. [En línea]. Available: https://moodle.org/plugins/qtype_geogebra. [Último acceso: 3 Julio 2019].

[17] Moodle, «Tipos de preguntas: Algebra,» 2019. [En línea]. Available: https://moodle.org/plugins/qtype_algebra. [Último acceso: 03 Julio 2019].

[18] Moodle, «Actividades: Contenido interactivo - H5P,» 2019. [En línea]. Available: https://moodle.org/plugins/mod_hvp. [Último acceso: 03 Julio 2019].

[19] H5P, «Ejemplos,» 2019. [En línea]. Available: https://h5p.org/content-types-and-applications. [Último acceso: 03 Julio 2019].

[20] Moodle, «Actividades: E-Voting,» 2019. [En línea]. Available: https://moodle.org/plugins/mod_evoting. [Último acceso: 03 Julio 2019].

[21] Moodle, «Actividades: Juego,» 2019. [En línea]. Available: https://moodle.org/plugins/mod_game. [Último acceso: 03 Julio 2019].

[22] J. M. Estrella Martínez, «El uso de Kahoot! en el proceso de aprendizaje en el aula. Una experiencia práctica en Ciclo Formativo de Grado Superior,» 10 Diciembre 2018. [En línea]. Available:

https://www.academia.edu/39688641/El_uso_de_Kahoot_en_el_p roceso_de_aprendizaje_en_el_aula._Una_experiencia_pr\%C3\%A 1ctica_en_Ciclo_Formativo_de_Grado_Superior. [Último acceso: 25 Julio 2019].

[23] M. B. Iván Sandoval, «Utilización de la modalidad aprendizaje combinado en el proceso de enseñanza aprendizaje de la asignatura fundamentos de matemática del curso propedéutico de la Escuela Politécnica Nacional,» 03 Noviembre 2016. [En línea]. Available: https://revistas.utp.ac.pa/index.php/clabes/article/view/983. [Último acceso: 25 Julio 2019].

[24] M. L. G. y. A. A. Benítez, «Competencias Matemáticas Desarrolladas en Ambientes Virtuales de Aprendizaje: el Caso de MOODLE,» 04 Abril 2011. [En línea]. Available: https://scielo.conicyt.cl/scielo.php?pid=S071850062011000300005\&script=sci_arttext\&tlng=en. [Último acceso: 25 Julio 2019].

[25] R. Maribe, Instructional Design: The ADDIE Aproach, Springer 2009 , pp. 3,12.

[26] I. E. Gámez, Los Modelos Tecno-educativos, revolucionando el aprendizaje del siglo XXI, 2014, p. 33

[27] U. o. Colorado, «PhET: ¿Qué es PhET?» 2019. [En línea]. Available: https://phet.colorado.edu/es/. [Último acceso: 14 Julio 2019].

[28] ThatQuiz, 2019. [En línea]. Available: https://www.thatquiz.org/. [Último acceso: 19 Julio 2019].

[29] C. World, 2019. [En línea]. Available: https://es.calcuworld.com/calculadoras-matematicas/. [Último acceso: 22 Julio 2019]. 